\title{
“Antena” de Fibra Óptica Plástica como Detectora em Enlaces LiFi
}

\author{
Juliana O. de Paula, Maristela Ciara, Ricardo M. Ribeiro, Vinicius N. H. Silva e Cláudia B. Marcondes
}

Resumo-Este artigo apresenta o desenvolvimento de um dispositivo que funciona como um detector de luz na faixa visível "antena óptica". A idéia central é utilizar um pequeno segmento de fibra óptica plástica de PMMA ou de poliestireno fluorescente para recepção em enlaces LiFi (Light Fidelity).

Palavras-Chave-comunicações por luz visível; fibra óptica plástica; espalhamento; fluorescência.

Abstract-This paper presents the development of a device that works as a light detector in the visible spectrum, like an "optical antenna". The idea is to use a small strand of plastic optical fiber made from PMMA or fluorescent polystyrene in a LiFi-link (Light Fidelity) receiver.

Keywords - visible light communications; plastic optical fiber; scattering; fluorescence.

\section{INTRODUÇÃO}

Uma multiplicidade de dispositivos, sensores e equipamentos vem sendo conectados em rede, ou Internet das Coisas (IoT) [1], o que causará um aumento na demanda por banda. Dessa forma, com a densificação, a alta demanda resultante deverá causar saturação no espectro de rádio, além de problemas de interferência. Bandas requeridas na faixa de $\mathrm{Gb} / \mathrm{s}$ para cada dispositivo, deverão demandar a tecnologia VLC (Visible Light Communications) ou LiFi (Light Fidelity) [2], que tem sido bastante explorada nos últimos anos com o intuito de complementar o WiFi (Wireless Fidelity) [3].

O LiFi é um sistema de comunicação que utiliza a luz visível como portadora. Devido aos requisitos de segurança ocular, esses sistemas utilizam quase que obrigatoriamente LEDs ao invés de LDs (Laser Diodes) como fontes ópticas. É um sistema que não requer licenciamento de espectro e sofre pouca influência de efeitos atmosféricos, como a turbulência nos sistemas FSO [4]. Porém, o LiFi é afetado pela mobilidade dos transceptores [5], sendo extremamente importante o aumento do campo-de-visão afim de atenuar erros de apontamento, o que causa aumento da taxa de erro de bit e até interrupção da comunicação.

Este trabalho supõe como cenário de aplicação real, um enlace LiFi entre um transmissor óptico fixo a LED e um detector óptico móvel posicionado em até cerca de $1 \mathrm{~m}$ de distância. O detector, consiste de um pequeno segmento (poucos centímetros) de fibra óptica plástica (POF) de PMMA ou fluorescente (FPOF), funcionando como uma espécie de "antena óptica" conectada à um fotodiodo para aumentar o campo-de-visão do mesmo. Desta forma, são descritos resultados experimentais comparativos de captura óptica em $\mathrm{dBm}$ do presente detector-LiFi.

Juliana O. de Paula, Maristela Ciara e Ricardo M. Ribeiro, Departamento de Engenharia de Telecomunicações Universidade Federal Fluminense, Niterói, RJ, Brasil, juliana_oliveira@id.uff.br; maristelaciara@id.uff.br e rmribeiro@id.uff.br. Vinicius N. H. Silva, Instituto de Telecomunicações, Coimbra, Portugal, vinicius.silva@ci.it.pt e Cláudia B. Marcondes, Coordenação de Telecomunicações, CEFET - RJ - Maracanã, Rio de Janeiro, RJ, Brasil, claudia.marcondes@cefet-rj.br.

\section{O DETECTOR LIFI: CAPTURA DE LUZ}

A Figura 1 mostra esquematicamente uma montagem experimental simples, destinada a medir o poder de captura de luz de alguns tipos de "antenas ópticas" plásticas a $90^{\circ}$ da direção do feixe luminoso. Está destacado por um retângulo pontilhado, o desenho esquemático do detector-LiFi, que consiste essencialmente de uma POF com $1 \mathrm{~mm}$ de diâmetro envolta por um tubo capilar de vidro de diâmetro interno 1,10 $\mathrm{mm}$ e comprimento $75 \mathrm{~mm}$ para uma melhor sustentação da fibra. Destacado em amarelo, temos a emenda entre a "POFantena" e um pequeno segmento de POF-PMMA feita com SuperBonder em gel, proporcionando uma perda por inserção $\mathrm{de} \sim 0,4 \mathrm{~dB}$ [6]. O segmento de POF-PMMA é acoplado à um fotodiodo que faz parte de um medidor de potência óptica (MPO) com $-50 \mathrm{dBm}$ de sensibilidade.

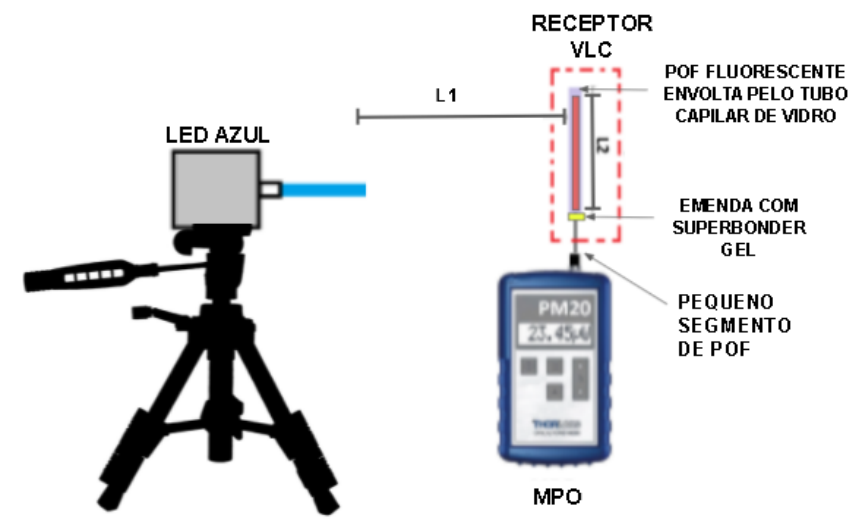

Fig. 1. Diagrama esquemático da montagem experimental. O retângulo pontilhado destaca o detector-LiFi.

As medidas foram divididas em 2 ciclos. O $1^{\circ}$ ciclo, a nível comparativo, serviu para avaliar qual tipo de POF mostra maior poder de captura de luz onde as "antenas" eram simplesmente inseridas no conector de entrada do MPO, sem o invólucro de vidro. $\mathrm{O} 2^{\circ}$ ciclo como mostra a Fig. 1, foi o de caracterização da POF de melhor resultado no $1^{\circ}$ ciclo, incluindo aprimoramentos nas emendas. Quatro tipos distintos de "antenas ópticas" foram caracterizadas no $1^{\circ}$ ciclo: $1^{\mathrm{a}}$ ) POF in natura, $2^{\mathrm{a}}$ ) POF com ranhuras feitas "a quente", $3^{\text {a }}$ ) POF com ranhuras feitas "a frio" e $4^{\mathrm{a}}$ ) FPOF (poliestireno fluorescente vermelho) modelo IF-810087 da Industrial Fiber Optics (USA). Em todos os quatro casos, a cobertura (opaca) protetora de polietileno foi cuidadosamente retirada. A idéia foi a de conseguir guiar pela POF no sentido do fotodiodo, uma máxima fração de potência óptica incidente externamente, esta última gerada por um LED azul posicionado perpendicularmente à fibra (Fig.1). O comprimento físico das "antenas ópticas" foi de $\mathrm{L}_{2}=5 \mathrm{~cm}$ nos 2 ciclos. Uma mesma lâmina metálica, tanto na temperatura ambiente quanto aquecida na mesma temperatura de um ferro de soldar comum $\left(\sim 180{ }^{\circ} \mathrm{C}\right)$, foi utilizada para imprimir ranhuras nas "antenas ópticas" a "frio" e a "quente", respectivamente. As ranhuras foram feitas tangencialmente e de forma superficial com periodicidade de $\sim 1 \mathrm{~mm}$ ao longo de $\mathrm{L}_{2}$. A "frio", estima-se 
ranhuras gravadas com profundidade de $0,1 \mathrm{~mm}$ enquanto que a "quente", $\sim 0,2 \mathrm{~mm}$. A idéia das ranhuras, foi a de aumentar o poder espalhador da POF através do mecanismo de espalhamento Mie, aumentando o poder de acoplamento luminoso [7]. Todas as medidas foram realizadas em ambiente escuro com o MPO devidamente ajustado para mostrar medidas calibradas em $460 \mathrm{~nm}$ (POF-PMMA) ou $635 \mathrm{~nm}$ (FPOF-vermelha). A razão da emenda da "POFantena" com a POF-PMMA, foi a de expor todo o comprimento $\mathrm{L}_{2}$ ao campo luminoso externo minimizando a re-absorção da fluorescência na FPOF [8], se for o caso.

O LED azul ( $460 \mathrm{~nm})$ com potência óptica de saída nominalmente em $4 \mathrm{~mW}$ @20 mA foi escolhido para a excitação da "antena óptica" pelos seguintes motivos: $1^{\circ}$ ) O espalhamento Rayleigh é mais intenso quanto menor for o comprimento de onda e $2^{\circ}$ ) No caso da FPOF, obtém-se maior potência fluorescente via excitação com luz azul [8], o que pode-se também obter com LEDs brancos, amplamente utilizados no LiFi. Uma desvantagem no $1^{\circ}$ caso, é a menor responsividade no azul exibida pelos fotodiodos de silício quando se compara com maiores comprimentos de onda. Conforme a Fig.1, a distância entre o transmissor e o receptor-VLC foi fixada em $\mathrm{L}_{1}=10 \mathrm{~cm}$. As Tabelas 1 e 2 mostram comparativamente os níveis de potência luminosa $(\mathrm{dBm})$ produzidos pelas "antenas ópticas", conforme as leituras no MPO nos ciclos 1 e 2, respectivamente.

Da Tabela 1, nota-se a baixa eficiência de captura da POF in natura que ficou no nível low signal. Como o MPO possui sensibilidade de $-50 \mathrm{dBm}$, é estimado um aumento na eficiência de captura de pelo menos $-36,9-(-50)=+13,1 \mathrm{~dB}$ gerada pelas antenas com ranhuras. Pode-se também notar que não fez diferença significativa realizar ranhuras "a frio" ou "a quente". Entretanto, a FPOF-vermelha apresentou um poder de captura $\sim 6 \mathrm{~dB}$ maior quando se compara com qualquer das POFs-PMMA com ranhuras. Com a FPOFvermelha, deve-se notar que ocorre uma conversão de comprimento de onda de $460 \mathrm{~nm}$ para $635 \mathrm{~nm}$. Um fotodiodo de Si possui maior responsividade em $635 \mathrm{~nm}$ quando se compara com $460 \mathrm{~nm}$, o que contribui para a referida melhora.

TABELA 1. Resultados comparativos do nivel de sinal óptico Pout gerado pelas "antenas ópticas" a $10 \mathrm{~cm}$ de distância do LED azul emissor no ciclo 1 .

\begin{tabular}{|c|c|c|}
\hline "Antena" óptica & Pout (dBm) & $\lambda(\mathrm{nm})$ \\
\hline PMMA in natura & Low signal & 460 \\
\hline PMMA c/ ranhuras a quente & $-36,9$ & 460 \\
\hline PMMA c/ ranhuras a frio & $-36,9$ & 460 \\
\hline FPOF-vermelha & $-31,0$ & 635 \\
\hline
\end{tabular}

Melhorias como mostra o retângulo pontilhado da Figura 1, foram realizadas na estrutura da antena. Então foi realizado o ciclo 2 de medições. A Tabela 2 indica os resultados dessas medições, porém obtidas somente com a FPOF-vermelha in natura ou com ranhuras.
TABELA 2. Resultados comparativos do nível de sinal óptico Pout gerado pelas "antenas ópticas" FPOF-vermelhas a $10 \mathrm{~cm}$ de distância do LED azul emissor no ciclo 2.

\begin{tabular}{|c|c|c|}
\hline $\begin{array}{c}\text { "Antena óptica": FPOF- } \\
\text { vermelha }\end{array}$ & Pout $(\mathrm{dBm})$ & $\lambda(\mathrm{nm})$ \\
\hline in natura & $-15,8$ & 635 \\
\hline c/ ranhuras a quente & $-14,9$ & 635 \\
\hline c/ ranhuras a frio & $-14,6$ & 635 \\
\hline
\end{tabular}

\section{CONCLUSÕES}

Pode-se concluir preliminarmente sobre a possibilidade de usar uma POF-PMMA comum com ranhuras superficiais como forma de aumentar o seu poder de espalhamento óptico e então a sua eficiência de captura luminosa. Porém, a FPOF-vermelha foi a que exibiu o melhor resultado. Além disto, pode-se observar que POFs fluorescentes riscadas superficialmente exibiram uma eficiência melhorada de $1,2 \mathrm{~dB}$ com relação a mesma fibra in natura. Técnicas para aumentar a captura de luz via espalhamento e/ou fluorescência devem ser mais a fundo investigadas. Porém neste trabalho, do low signal onde $<-$ $50 \mathrm{dBm}$ até $-14,6 \mathrm{dBm}$ com a FPOF-vermelha riscada a frio, obteve-se uma melhora na captura de luz de pelo menos $-14,6 \mathrm{dBm}-(-50 \mathrm{dBm})=+35,4 \mathrm{~dB}$ em condições experimentais bastante similares.

Embora não tenha sido aqui mostrado, a "antena óptica" receptora com FPOF, respondeu temporalmente à incidência de um tom senoidal modulando o LEDtransmissor em pelo menos até $10 \mathrm{MHz}$.

\section{AgRADECIMENTOS}

Autores agradecem a Coordenação de Aperfeiçoamento de Pessoal de Nível Superior (CAPES - Finance Code 001), Conselho Nacional de Desenvolvimento Científico e Tecnológico (CNPq) e Fundação de Amparo à Pesquisa do Estado do Rio de Janeiro (FAPERJ).

\section{REFERÊNCIAS}

[1] Demirkol, I., Camps-Mur, D., Paradells, J., Combalia, M., Popoola, W. \& Haas, H. (2019). Powering the internet of things through light communication. IEEE Communications Magazine, 57(6), 107-113.

[2] Burchardt, H., Serafimovski, N., Tsonev, D., Videv, S., \& Haas, H. Communications Magazine, 52(7), 98-105.

[3] A. Sarkar, S. Agarwal and A. Nath, "LiFi Technology: Data Transmission through Visible Light," Int. J. of Advance Research in Computer Science and Management Studies, vol. 3, issue 6, pp. 1-12, June 2015.

[4] De Oliveira G, Silva VN, Barbero AP, Ribeiro RM, Coelho TV, Dos Santos AB. Measurements of indoor/outdoor atmospheric turbulence through optical triangulation method. Measurement Science and Technology. 2017 Apr 6;28(5):055207.

[5] A. C. Boucouvalas, P. Chatzimisios, Z. Ghassemlooy, M. Uysal and K, Yiannopoulos, "Standards for Indoor Optical Wireless Communications," IEEE Communications Magazine - Communications Standards Supplement, pp. 24-31, March 2015.

[6] Michele C. Zanon, Vinicius N. H. Silva, Andrés P. L. Barbero and Ricardo M. Ribeiro, Practical splicing of poly-methyl-methacrylate plastic optical fibers, Applied Optics, 57,4, 812-816, February 2018.

[7] M. Poppendieck and D. Brown, "Control of Light Output from Plastic Optical Fiber with Optical Elements," pp. 580-584, 1996.

[8] Ribeiro, R. M., Silva, V. N. H., Barbero, A. P. L., Alves, C. M., \& Rodrigues, C. R. L. (2015). Fast wavelength conversion to generate 560 $\mathrm{nm}$ fluorescence for data transmission in polymer optical fibers. Electronics Letters, 51(2), 168-170. 